\title{
Surgical treatment of condylar head fractures, the way to minimize the postraumatic TMJ ankylosis
}

\author{
Hirjak D ${ }^{1}$, Machon $\mathrm{V}^{2}$, Beno $\mathrm{M}^{1}$, Galis $\mathrm{B}^{1}$, Kupcova $\mathrm{I}^{1}$ \\ Department of Oral and Maxillofacial Surgery, Comenius University, University Hospital Ruzinov, \\ Bratislava, Slovakia. hirjak.dusan@gmail.com
}

\begin{abstract}
Management of condylar head fractures (CHF) of the temporomandibular joint (TMJ) remains a source of controversy. Based on established literature, group of CHFs and conservative treatment connected with period of maxillomandibular fixation (MMF) increases the risk for TMJ ankylosis. This paper presents anatomical and functional results of surgical treatment of condylar head fractures in a group of 24 patients (29 joints). Fractures were diagnosed based on conventional radiographs and computed tomography (CT) scans. Utilising an intraoperative arthroscopy authors evaluated actual intraarticular posttraumatic changes. This study presents acceptable functional and radiological results of surgical treatment of condylar head fractures with more than 3-year follow-up. The authors believe that re-establishing the pretraumatic anatomic position of the TMJ components (fragment and the disc) and early postoperative rehabilitation are inevitable to minimize the risk of postraumatic TMJ ankylosis (Tab. 2, Fig. 8, Ref. 31). Text in PDF www.elis.sk.

KEY WORDS: condylar head fracture, articular disc, intraoperative arthroscopy, ankylosis.
\end{abstract}

\section{Introduction}

Mandibular fracture represents the most common facial bone injury $(57 \%)(11,23,30)$. Mandibular condylar fractures account for between $21 \%$ and $52 \%$ of all mandibular fractures $(11,23,30)$. The treatment of mandibular condyle fractures is still controversial. There is no general consensus with regard to which fractures should be treated by closed or open approach $(3,21,25)$. Condyloid process fractures have been managed by closed techniques with a period of maxillomandibular fixation (MMF) or functional therapy using guiding elastics to maintain proper occlusion. Many surgeons favor open reduction and internal fixation (ORIF) because it provides anatomical reduction of the fragments, rapid healing and early restoration of function (29, 30). Clinically condylar head fractures (CHF) were not commonly detected on conventional radiographs. With introduction of computed tomography (CT), the incidence of detection of such fractures increased and was reported to be 9-29\% of all condylar fractures (19). Historically, condylar head fractures were treated by closed reduction using maxillomandibular fixation (MMF) because immediate satisfactory results were easily attainable. The small fractured fragment was difficult to reduce and fix and there was a fear of facial nerve

${ }^{1}$ Department of Oral and Maxillofacial Surgery, Comenius University, University Hospital Ruzinov, Bratislava, Slovakia, and ${ }^{2}$ Department of Stomatology, Ist Faculty of Medicine, Charles University, General University Hospital, Prague, Czech Republic

Address for correspondence: D. Hirjak, MD, PhD, Department of Oral and Maxillofacial Surgery, Comenius University, University Hospital Ruzinov, Ruzinovska 6, SK-826 06 Bratislava, Slovakia. injury $(10,24,30)$. Complications, such as temporomandibular joint (TMJ) dysfunction, osteoarthrosis, pain and ankylosis must be considered when using closed treatment. Many studies have shown that ankylosis mostly occured in patients with condylar head fractures $(1,2,7)$. The most important etiological factors of ankylosis are destruction of the condylar head, articular disc and lateral pterygoid muscle resulting in restriction of mouth opening (31). In order to determine the effect of the injury on the articular disc and condyle in CHF cases, authors evaluated intraarticular structures, articular disc position and function arthroscopically. They did not find any disc rupture or other disc injury. Hyperemia of retrodiscal tissue, hyperemia and multiple haemoragies of medial capsule and dislocation of the disc with elongation of retrodiscal tissue were seen arthroscopically. The aim of open surgery is to reestablish the pre-traumatic anatomic relations of the fragments to the condyle and the disc to prevent further injury to the disc. On the other hand the closed reduction treatment followed by active and intensive functional therapy due to movement of the sharp ramus stump may increase traumatic changes like tearing and perforation of the disc and lateral capsule. Post-traumatic intracapsular bleeding may potentially results in restricted mouth opening and create fibrous and bony ankylosis in the future.

The purpose of this study is to present outcomes of ORIF of CHFs focused on fragment reposition and fixation and the TMJ function minimum 3 years postoperatively.

\section{Patients and methods}

From 2006 to 2012 (7 years), 204 patients (231 joints) were diagnosed with mandibular condyle fracture. 24 patients ( 29 joints, 
$12.5 \%$ ) had condylar head fractures and all of them were treated by open surgery. We excluded from our study group edontulous patients, noncooperative patients, patients with associated maxillary fractures, zygomatic fractures and patients with multiple and comminuted mandibular fractures because of the difficulty with postoperative functional therapy.

Patients had obvious symptoms preoperatively, such as limitation of mouth movement, malocclusion, and lateral deviation of the mandible during opening. There were 20 unilateral and 4 bilateral fractures. In 5 cases there were concomitant fractures of of the mandible (symphysis 3, contralateral angulus 2). All the fractures had a fracture line typically going from superolaterally to inferomedially Fractures were classified using Neff's classification (Type A: 2 fractures, Type B: 20 fractures, Type C: 7 fractures) $(15,18)$. Bony fragments were always typically displaced infero-

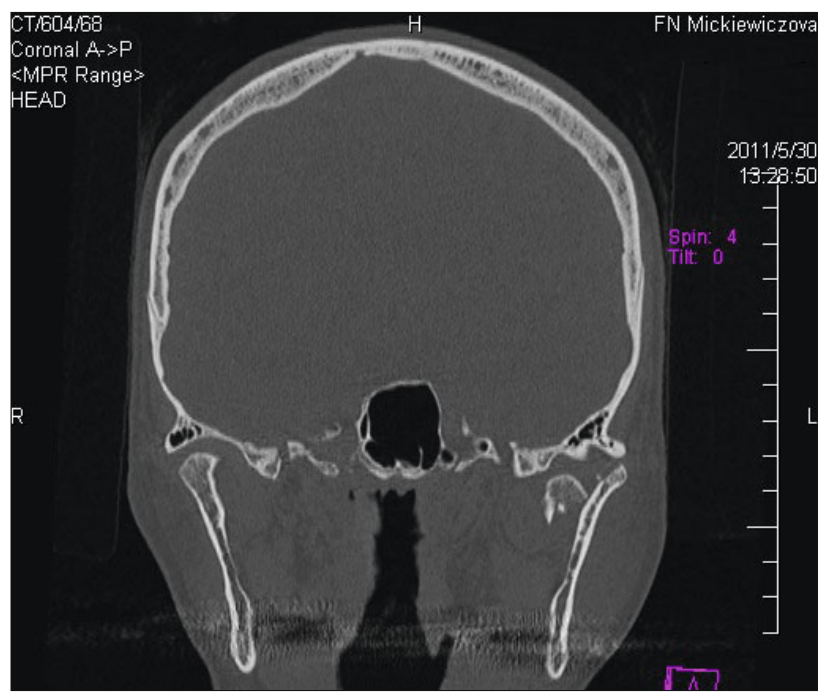

Fig. 1.CT scan, coronal view of typically dislocated fragment of the left CHF.

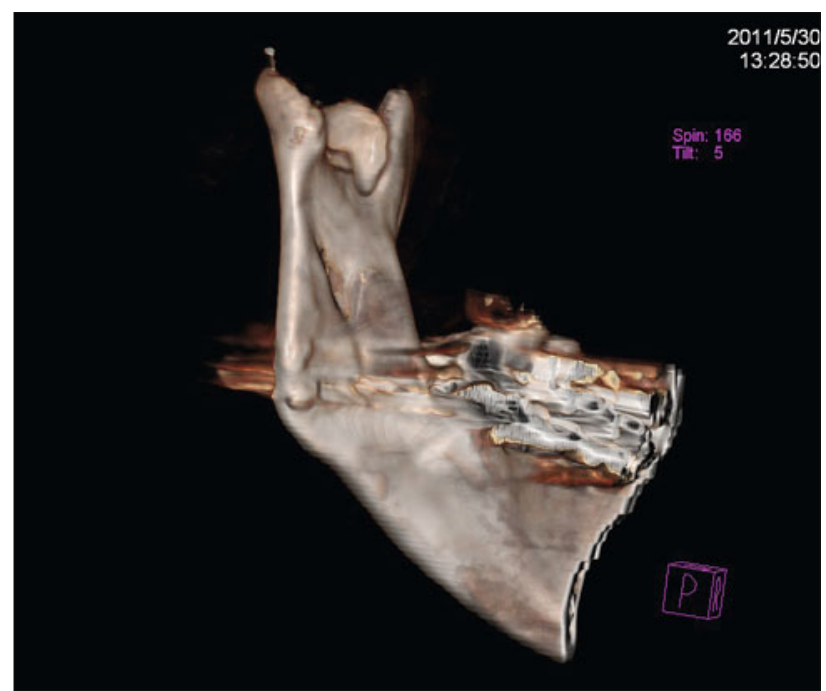

Fig. 2. 3-dimensional CT scan, medial view on dislocated fragment of the left CHF.

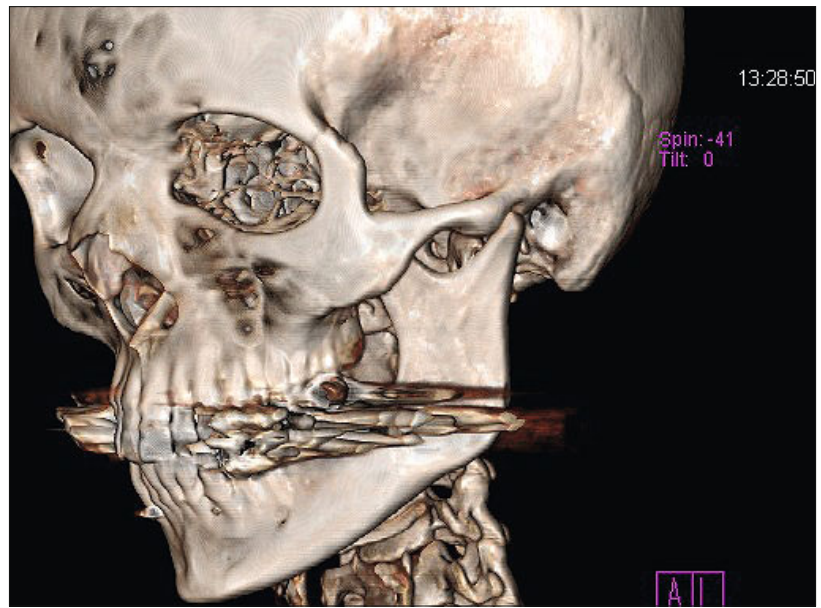

Fig. 3. 3-dimensional CT scan, leteral view on dislocated fragment of the left CHF.

medially. Fractures were diagnosed on the basis of conventional radiographs (orthopantomogram, posteroanterior radiogram) as well as CT scans in coronal and axial planes. In some cases to better determine the position of fragments we utilized 3-dimensional CT scanning (Figs 1-3).

Of the 24 patients, 10 were female and 14 were male. Mean age at the time of injury was 33.6 years (range 19-58 years) Mean follow-up period was 40.5 months (range 37.5 months to 43 months). The mean delay between injury and surgery was 6.2 days. Most frequent cause was interpersonal violence in 10 patients (42\%), sport (bicycle, football) in 6 patients (25\%), traffic accident in 3 patients (12\%), and fall in 5 patients (21\%).

\section{Surgical procedure}

The preauricular approach was used for open treatment. The zygomatic arch, articular eminence and the TMJ capsule were exposed after elevation of the skin flap. Before opening the lower joint compartment and identifying the fragments an arthroscope (KARL STORZ endoscope $1.9 \mathrm{~mm}$, Tuttlingen, Germany) was introduced to the upper joint compartment. The aim of arthroscopy was to assess the intraarticular structures, position, and function of the articular disc. The upper joint compartment was evaluated from posterior disc attachement to anterior recess (Figs 4 and 5).
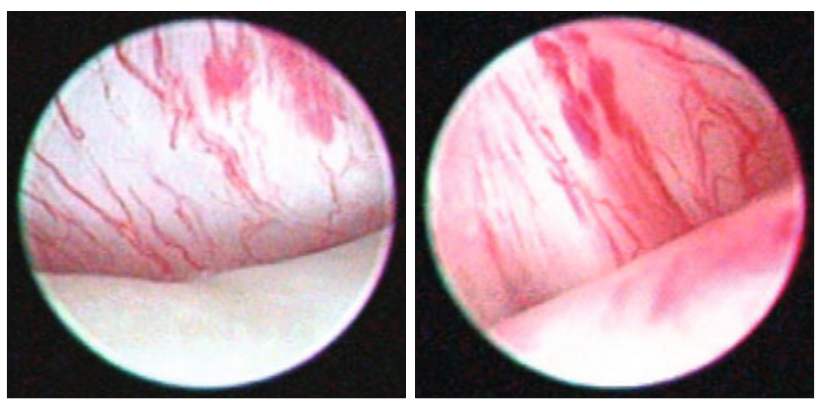

Fig. 4., 5. Perioperative arthroscopy of the TMJ, vascularisation and haemorrhagic changes. 


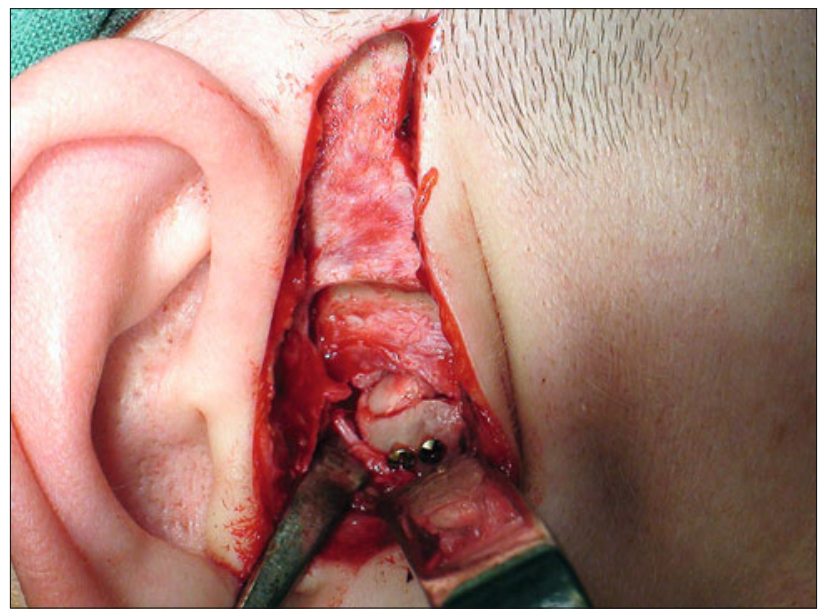

Fig. 6. Preauricular surgical approach, fragments fixed using 2 screw.

A t-shaped incision was made to open the capsule of the TMJ. The stump of condylar fracture was exposed. Sigmoid notch was exposed and condylar fragment was found displaced anteromedially and inferiorly. The displaced bony fragment was indentified and using blunt periosteal elevator, the displaced bony fragment and the disc were reduced into the correct position and relationship.

Care must be taken when repositioning the displaced bone fragment to avoid striping its soft tissue connection or damaging the articular cartilage. In this phase, the patient should be in a state of complete relaxation. Inferior traction on mandibular angle is very important to widen the joint space. Fixation of bone fragments was performed using self-tapping screws ( $1.5 \mathrm{~mm}$ with 12 to 16 $\mathrm{mm}$ of lenght). 2 screws are recommended for better stability of fragments (Fig. 6). In some cases the compact bone is too thin so a microplate (BIOMET Microfixation, $1.5 \mathrm{~mm}$, Jacksonville, FL) was used to fixate the bone fragment. TMJ function and occlusion were checked and the wound was closed in layers. Guiding elastics were left in place for 5 to 10 days, depending on occlusion.

The patients were placed on a liquid diet for 6 weeks. Control orthopantomogram (OPG) and posteroanterior radiogram of the skull were performed 7 days postoperatively. Patients were followed 1 week, 1 month, 3 months, 6 months and 1 year postoperatively. Control CT imaging was performed 1 month and 1 year and 3 years postoperatively.

\section{Results}

In total, 23 patients (28 joints) were followed-up, 1 patient was excluded because he did not return for follow-up. In general, the treatment outcomes were successful in all the patients in our study group. Occlusion was not altered in any case, either objectively and/or subjectively. Clicking on mouth opening was noted in 3 joints (11\% ) 6 months postoperatively. 1-month postoperative CT scans showed absolute anatomic reduction in 13 joints ( $46 \%)$, near anatomic reduction in 12 joints (43\% ) and moderate reduction with slight ramus shortening in 3 joints (11\%). There was no imaging evidence of bone resorbtion or necrosis of the condy-

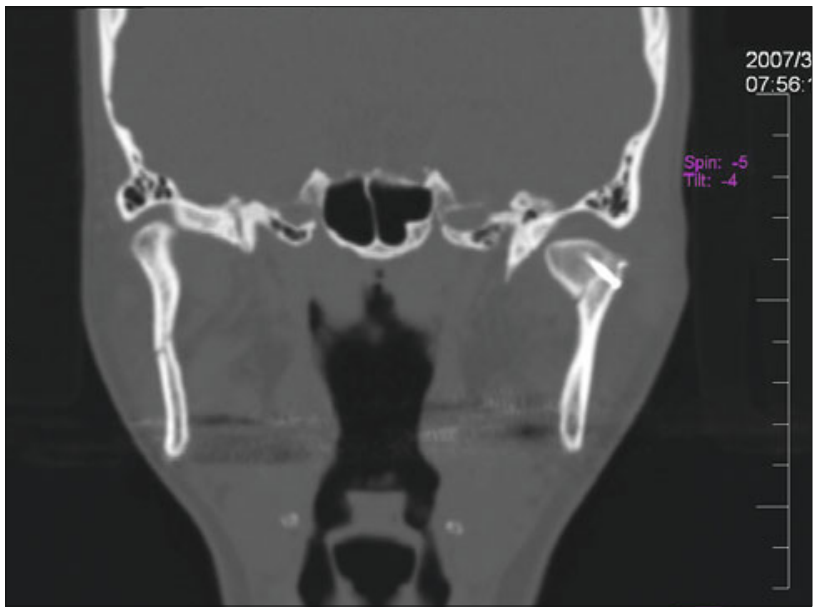

Fig. 7. CT scan, coronal view on repositioned and fixed left CHF.

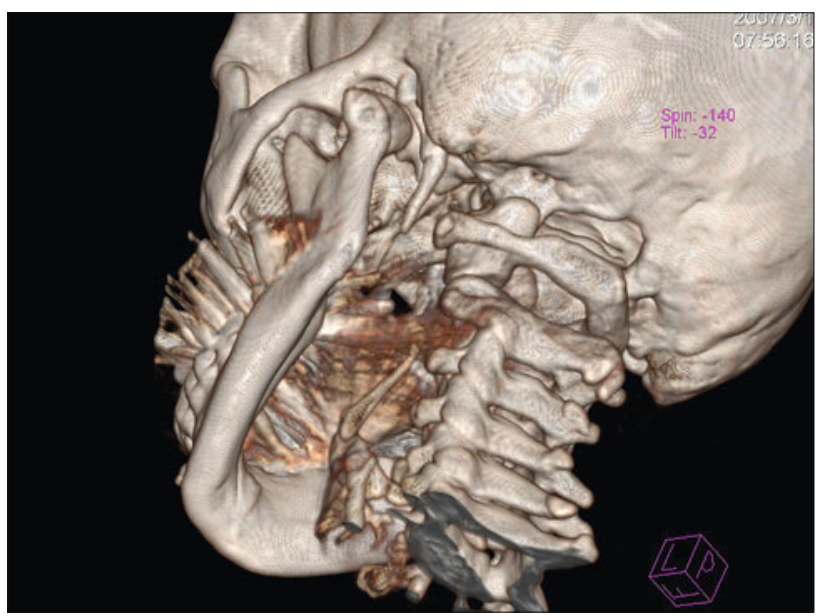

Fig. 8. 3-dimensional scan, lateral view on repositioned and fixed left CHF.

lar head on CT scans 1and 3 years postoperatively (Figs 7 and 8). There was no indication for plate or screws removal. Postoperative weakness of the temporal branch of the facial nerve was present in 3 patients and function regained in 5 months in all patients.

Distribution of the patients, age, gender, fracture side, concomitant fracture of the mandible and type of fragment fixation are shown in Table 1. Anatomical position of the fragments, function and post treatment deviation during opening are shown in Table 2. Clinical examination showed that mean maximal post treatment interincisal mouth opening was $40.1 \mathrm{~mm}$ (range 28 - $52 \mathrm{~mm}$ ). Mean post treatment lateral deviation of the mandible during opening was $2 \mathrm{~mm}$ (range $0-4 \mathrm{~mm}$ ).

\section{Discussion}

Our data showed that condylar head fractures (CHF) account for $12.5 \%$ of all condylar fractures treated surgicaly. The treatment of CHFs (intracapsular, diacapitular, sagittal fractures) has been debated over recent years, and indications for open reduction and internal fixation compared with closed reduction remain 
Tab. 1. Distribution of the patients, age, gender, fracture side, concomitant fracture, type of fixation.

\begin{tabular}{|c|c|c|c|c|c|c|c|c|c|}
\hline \multirow[t]{2}{*}{ Patient } & \multirow[t]{2}{*}{ Age } & \multicolumn{2}{|c|}{ Gender } & \multicolumn{2}{|c|}{ Fracture side } & \multirow[t]{2}{*}{$\begin{array}{c}\text { Concomitant } \\
\text { mand. fr. }\end{array}$} & \multicolumn{3}{|c|}{ Type of fixation } \\
\hline & & $\mathrm{F}$ & $\mathrm{M}$ & uni. l. & bilat & & 2 screws & miniplate & combination \\
\hline 1. & 21 & & 1 & 1 & & & 1 & & \\
\hline 2. & 29 & 1 & & 1 & & & 1 & & \\
\hline 3. & 58 & 1 & & 1 & & & & 1 & \\
\hline 4. & 35 & 1 & & 1 & & & & 1 & \\
\hline 5. & 54 & & 1 & 1 & & & 1 & & \\
\hline 6. & 29 & & 1 & 1 & & & & 1 & \\
\hline 7. & 44 & 1 & & 1 & & & 1 & & \\
\hline 8. & 21 & & 1 & 1 & & symphysis & & 1 & \\
\hline 9. & 41 & & 1 & 1 & & & 1 & & \\
\hline 10. & 35 & & 1 & 1 & & & 1 & & \\
\hline 11. & 34 & & 1 & 1 & & & & & 1 \\
\hline 12. & 23 & 1 & & & 1 & angulus & 1 & & \\
\hline 13. & 28 & 1 & & 1 & & & & 1 & \\
\hline 14. & 20 & & 1 & 1 & & & 1 & & \\
\hline 15. & 27 & & 1 & 1 & & & & 1 & \\
\hline$\overline{16 .}$ & 52 & & 1 & 1 & & symphysis & & 1 & \\
\hline 17. & 50 & 1 & & 1 & & & & & 1 \\
\hline 18. & 39 & & 1 & & 1 & & & 1 & \\
\hline 19. & 29 & 1 & & & 1 & symphysis & 1 & & \\
\hline 20. & 35 & 1 & & 1 & & & 1 & & \\
\hline 21. & 27 & 1 & & & 1 & & & & 1 \\
\hline 22. & 34 & & 1 & 1 & & angulus & & 1 & \\
\hline 23. & 19 & & 1 & 1 & & & 1 & & \\
\hline
\end{tabular}

Tab. 2. Position of the fragments, postoperative TMJ function.

\begin{tabular}{|c|c|c|c|c|c|c|c|c|}
\hline \multirow[t]{2}{*}{ Patient } & \multicolumn{3}{|c|}{ post op. position of the fragment } & \multicolumn{5}{|c|}{ Function } \\
\hline & $\mathrm{A}$ & $\mathrm{N}$ & $\mathrm{M}$ & MMO & LA & $\mathrm{LN}$ & $\mathrm{PR}$ & DV \\
\hline 1. & & 1 & & 42 & 14 & 11 & 6 & 4 \\
\hline 2. & 1 & & & 41 & 9 & 8 & 5 & 2 \\
\hline 3. & 1 & 1 & & 42 & 12 & 8 & 7 & 4 \\
\hline 4. & & & 1 & 48 & 10 & 7 & 6 & 2 \\
\hline 5. & 1 & & 1 & 42 & 11 & 6 & 4 & 3 \\
\hline 6. & & 1 & & 36 & 8 & 5 & 4 & 3 \\
\hline 7. & & 1 & & 35 & 7 & 5 & 4 & 4 \\
\hline 8. & & 1 & & 52 & 11 & 11 & 8 & 0 \\
\hline 9. & 1 & & & 43 & 7 & 5 & 4 & 3 \\
\hline 10. & 1 & & & 47 & 9 & 6 & 5 & 4 \\
\hline 11. & & & 1 & 32 & 7 & 5 & 4 & 3 \\
\hline 12. & & 2 & & 46 & 10 & 10 & 7 & 1 \\
\hline 13. & & & 1 & 40 & 9 & 6 & 4 & 3 \\
\hline 14. & 1 & & & 28 & 7 & 5 & 4 & 4 \\
\hline 15. & 1 & & 1 & 30 & 8 & 5 & 6 & 4 \\
\hline 16. & 1 & & & 35 & 8 & 5 & 4 & 4 \\
\hline 17. & 1 & & & 36 & 8 & 5 & 4 & 3 \\
\hline 18. & & 2 & & 52 & 11 & 8 & 4 & 1 \\
\hline 19. & 2 & & & 42 & 7 & 9 & 3 & 0 \\
\hline 20. & 1 & & & 38 & 9 & 6 & 4 & 1 \\
\hline 21. & & 1 & & 39 & 7 & 6 & 3 & 1 \\
\hline 22. & & 1 & & 36 & 8 & 7 & 3 & 0 \\
\hline 23. & 1 & & & 41 & 9 & 6 & 4 & 1 \\
\hline
\end{tabular}

A - absolute anatomic reduction, $\mathrm{N}$ - near anatomic reduction, $\mathrm{M}$ - moderate reduction, $\mathrm{MMO}$ - maximum mouth opening, LA - lateral pulsion on affected side, LN lateral pulsion on unaffected side, $\mathrm{PR}$ - propulsion, DV - deviation at maximal opening (all in mm

controversial (3, 9, 15, 20, 21). Non-surgical methods are widely accepted for treating children with sagittal condylar fractures but no consensus has been established for adult patients. However, it appears that more maxilofacial surgeons favour open reduction and internal fixation rather than closed reduction in the treatment of $\mathrm{CHF}$

Traumatic lesions of TMJ may lead to severe TMJ disorders, osteoarthrosis of articular surfaces and even ankylosis. Arakeri and 
others $(1,2,7,16)$ published observation that sagittal fractures of articular head can be the cause of ankylosis.

Resorption of the condyle postoperatively is possible because of changes in the condyle's position, presence of osteosynthetic alloplastic material and increasing functional loading $(4,10,18)$. The surgeon should ensure that anatomic reduction of the fracture fragments and disc occured to avoid destruction of the capsule and lateral pterygoid muscle. Several techniques have been proposed for surgical fixation of condylar head fragments. Rasse (26) discussed the benefit of resorbable pins from an auricular access. Kermer et al (13) described technique using two miniscrews. Vesnaver (29) described the benefit of using lag screws. Pilling et al (25) described a variation and benefit of the cannulated lag screws.

Each osteosynthesis device inserted into the condylar head may compromise the blood supply. Authors recommend to use 2 self-tapping microscrews $(1.5 \mathrm{~mm})$ for fixation of condylar head fracture fragments. This method increases the stability of fixation for the condylar head with high amount of spongy bone. One long screw parallel to the condylar long axis is not stable enought for fragment fixation (7). We do not utilize retrieval of the displaced condylar fragment with a screw (19). Using two periosteum blunt elevators and relaxation of the patients is safe enough to reach precise anatomical adaptation of the fragments and the disc.

In condylar head fractures, specially at type B, C of the Neff classification $(17,20)$ the traction of lateral pterygoid muscle dislocates the fragment anteromedially and inferiorly sometimes resulting in shortening of the posterior ramal vertical height. This may cause restriction of the mandibular movement and malocclusion. Posttraumatic dislocation of the bony fragment of the condyle can be associated with serious complications, restricted mouth opening, pain, including ankylosis. Using conservative (non-surgical) treatment, anatomical reduction of condylar fragment in CHF cannot be typically achieved. In such cases, active and agressive physiotherapy allows the condyloid process stump to articulate with the articular disc interposed between. This sharp condylar stump traumatizes the disc itself and sensitive bilaminar zone of dislocated disc causing pain and reflectoric muscle contraction. Related complications, such as malocclusion, asymmetry, internal derangement, joint pain, locking and joint degeneration, even ankylosis may not be initially apparent but can develop later. The aim of treatment should be reestablishment of the pretraumatic anatomic disc position as well as anatomical shape and position of condylar head which are the basis for long term function.

Nussbaum et al (24) concluded in a meta-analysis of the treatment of condylar fractures that due to the variabilites of treatments discussed in literature, no prefered method, open or closed, could be recommended. Other studies such as those by Eckelt et al (3) and Hlawitschka and Eckelt(9) have shown superior results with the surgical option. Hlawitschka et al (10) reported poor functional and radiological results after closed treatment.

Despite controversy in management of condylar head fractures, most agree that ORIF provides better results long term. Authors are convinced that ORIF as described in this paper, especially in cases of condylar head fractures (Type B, C of Neff's classification) provides good functional results. This statement is supported by arthroscopicaly revealed pretraumatic status and position of the disc and condylar fragment and their precise reposition as demostrated in this study.

Function of the disc plays a keyrole in TMJ unrestricted movement $(2,4,5,12,16)$. In CHF, despite of the fact that the disc is usually stretched and displaced anteromedially there were no ruptures or another pathological changes seen in our series of cases. Hypervascularity and small bleedings at posterior attachment and medial joint capsule were present. In closed reduction of $\mathrm{CHF}$, the fractured condylar stump during intensive physical therapy will propably cause an additional tearing and bleeding or even perforation of the disc and posterior and lateral attachments. These anatomical changes coupled with pain may decrease mandibular movement and interrupt the mechanical barrier the disc provides and can provide the enviroment for future ankylosis. To avoid these pathological posttraumatic changes and avoid heterotopic bone formation is the goal of the precise anatomical repositioning of medial pole of the condyle together with re-establishment of its relationship to the articular disc $(1,2,7,10)$. From the ethical reasons it is not possible to make a randomized study in this controversial topic. Results presented in this study showed a good functional result in 3 year follow-up despite of a small number of patients.

In conclusion the function, occlusion and CT scans of the TMJ in 3 year follow-up showed acceptable results. While the results of this study are encouraging, larger, long-term randomized clinical trials are necessary.

\section{References}

1. Arakeri G, Kusanale A, Zaki GA. Pathogenesis of post-traumatic ankylosis of the temporomandibular joint: a crititcal review. Brit J Oral Maxillofac Surg 2011.

2. Duan DH, Zhang Y. A clinical investigation on disc displacement in sagital fracture of the mandibulare condyle and its association with TMJ ankylosis development. Int J Oral Maxillofac Surg 2011; 40: 134-138.

3. Eckelt U, Schneider M, Erasmus F et al. Open versus closed treatment of fractures of mandibular condylar process - A prospective randomized multi-centre study. J Craniomaxillofac Surg 2006: 34: 306-310.

4. Gerhard S, Ennemoser T, Rudisch A. Condylar injury: magnetic resonance imaging findings of temporomandibular joint soft-tissue changes. Int J Oral Maxillofac Surg 2007: 36: 214-218.

5. Goss AN, Bosanquet AG. The arthroscopic appearence of acutetemporomandibular trauma. J Oral Maxillofac Surg 1990; 48: 780-783.

6. Hackenberg B, Lee C, Caterson EJ. Management of subcondylar mandible fractures in the adult patient. J Craniofac Surg 2014; 25 (1): 166-171.

7. He D, Ellis EIII., Zhang Y. Etiology of Temporomandibular Joint Ankylosis Secondary to Condylar fractures: The role of concomitant mandibular fractures. J Oral Maxillofac Surg 2008; 66: 77-84.

8. He D, Yang Ch, Chen M, Jiang B. Intracapsular condylar fracture of the mandible: our clasification and open treatment experience. J Oral Maxillofac Surg 2009: 67: 1672-1679.

9. Hlawitschka M, Eckelt U. Assesment of patients treated for Intracapsular fractures of the mandibular condyle by closed techniques. J Oral maxillofac Surg 2002; 60: 784-791. 
10. Hlawitschka M, Loukota R, Eckelt U. Functional and radiological results of open and closed treatment of intracapsular (diacapitular) condylar fractures of the mandible. Int J Oral Maxillofac Surg 2005; 34: 257.

11. Chen CT, Feng CH, Tsay PK, Lai JP, Chen YR. Functional outcomes following surgical treatment of bilateral mandibular condylar fractures. Int J Oral Maxillofac Surg 2011; 40: 38-44.

12. Jones JK,Van Sickels JE. A preliminary report of arthroscopic findings following acute condylar trauma J Oral Maxillofac Surg 1991; 49: 55-60.

13. Kermer CH, Undt G, Rasse M. Surgical reduction and fixation of intracapsular condylar fractures. Int J Oral Maxillofac Surg 1998; 27: 191-194.

14. Kolk A, Neff A. Long-term results of ORIF of condylar head fractures of the mandible: A prospective 5-year follow-up study of small-fragment positional-screw osteosynthesis (SFPSO). J Cranio-Maxillofac Surg 2015; 43 (4): 452-461.

15. Landes Ca, Day K, Lipphardt R, Sader R. Closed versus open operative treatment of nondisplaced diacapitular fractures. J Oral maxillofac Surg 2008; 66: 1586-1594.

16. Laskin DM. Role of the meniscus in the etiology of posttraumatic temporomandibular joint ankylosis. Int J Oral Maxillofac Surg 1978; 7 (4): 340-345 .

17. Loukota RA, Neff A, Rasse M. Nomeclature/classification of the fractures of condylar head. Technical note. Br J Oral Maxillofac Surg 2010; 48: 477-478.

18. Merrill RG. Discussion: the arthroscopic appearence of acute temporomandibular trauma. J Oral Maxillofac Surg 1990; 48: 784.

19. Meng FW, Liu YP, Hu KJ, Kong L. Use of a temporary screw for alignment and fixation of sagittal mandibular condylar fractures with lateral with lateral screws. Int J Oral Maxillofac Surg 2010; 39: 548-553.

20. Neff A, Chossegros C, Blanc J-, Champsaur P, Cheynet F, Devauchelle B, Eckelt U, Ferri J, Gabrielli MFR, Guyot L, Koppel DA, Meyer C, Müller B, Peltomäki T, Spallaccia F, Varoquaux A, Wilk A, Pitak-Arnnop P. Position paper from the IBRA symposium on surgery of the head - the 2nd international symposium for condylar fracture osteosynthesis, marseille, france 2012. J Cranio-Maxillofac Surg 2016; 42 (7): 1234-1249.
21. Neff A, Kolk A, Deppe H et al. New aspect for indications of surgical management of intra-articular and hight temporomandibular dislocation fractures. Mund Kiefer Gesichtschir 1999; 3: 24.

22. Neff A, Kolk A, Neff F, Horch HH. Surgical vs. conservative therapy of diacapitular and high condylar fractures with dislocation. A comparison between MRI and axiography. Mund Kiefer Gesichtschir 2002; 4: 66-73.

23. Niezen ET, Bos RRM, DeBont LGM, Stegenga B. Complaints related to mandibular function impairment after closed treatment of fractures of the mandibular condyle. Int J Oral Maxillofac Surg 2010; article in press.

24. Nussbaum ML, Laskin DM, Best Am. Closed versus open reduction of mandibular condylar fractures in adults: a meta-analysis. J Oral Maxillofac Surg 2008; 66: 1087-1092.

25. Pilling E, Schneider M, Mai R, Loukota RA, Eckelt U. Minimally invasive fracture treatment with cannulated lag screws in intracapsular fractures of the condyle. J Oral Maxillofac Surg 2006; 64: 868-672.

26. Rasse M. New developments in the treatment of fractures of the temporomandibular joint. Mund Kiefer Gesichtschir 2000; 4: 69-87.

27. Schneider M, Loukota R, Eckelt U. Reduction of diacapitular fractures of the mandibular condyle using a special repositioning pin(technical note). Br J Oral Maxillofac Surg 2009; 47: 558-559.

28. Schneider M, Erasmus F, Gerlach KL, Kuhlisch E, Loukota RA, Rasse $\mathbf{M}$ et al. Open reduction and internal fixation versus closed treatment and mandibulomaxillary fixation of fractures of the mandibular condylar process: randomized, prospective, multicentric study with special evaluation of the fracture level. J Oral Maxillofac Surg 2008; 66: 2537-2544.

29. Vesnaver A. Open reduction and internal fixation of intra-articular fractures of the mandibular condyle: our first experiences. J Oral Maxillofac Surg 2008; 66: 2123-2129.

30. Villarreal PM, Monje F, Junguera LM, Mateo J, MorrilloAJ, Gonzales C. Mandibular condyle fracture: Determinant of treatment and outcome. J Oral Maxillofac Surg 2004; 62: 155-163.

31. Xiang G, Long X, Deng M, Han Q, Meng Q, Li B. A retrospective study of temporomandibular joint ankylosis secondary to surgical treatment of mandibular condylar fractures. Br J Oral Maxillofac Surg 2014; 52 (3): $270-274$. 